\title{
LA ESI COMO MOVIMIENTO PEDAGÓGICO EN EXPANSIÓN Y SUS TRANSFORMACIONES EPISTÉMICAS
}

\section{Comprehensive Sex Education as an Expanding Pedagogical Movement and Its Epistemic Transformations}

http://doi.org/10.33255/25914669/585

\section{Luciana Lavigne}

Facultad de Filosofía y Letras, Universidad de Buenos Aires

paoladogliottimoro@gmail.com https://orcid.org/0000-0002-8122-8941

Instituto de Investigaciones de Estudios de Género, Facultad de Filosofía y Letras, Universidad de Buenos Aires

jepechin2017 @gmail.com https://orcid.org/0000-0003-0262-520X

Recibido: 11 de octubre de 2020

Aceptado: 20 de abril de 2021

\section{Resumen}

A partir de la sanción de una ley nacional en 2006, la Educación Sexual Integral (ESI) se ha institucionalizado como política pública en Argentina. Diversas luchas culturales impregnaron este procesoy fueron redefiniendo las bases epistémicas de esta política educativa. En este trabajo, desde una

1 Este artículo retoma y profundiza un trabajo anterior de lxs autorxs en una nueva ruta reflexiva, por lo que algunos argumentos son reproducidos aquí para (re)situar la Educación Sexual Integral como plataforma institucional de un movimiento pedagógico que, en el escenario político del presente, está transformando intensamente las coordenadas sexo-genéricas de la escolaridad (Lavigne y Péchin, 2021). 
perspectiva etnográfica crítica, mostramos cómo se fue produciendo un movimiento pedagógico en torno a este proceso de construcción política, involucrando a movimientos sociales, activismos políticos y organismos del estado, que, en articulación con cambios legislativos que implicaron una democratización de la ciudadanía, promovieron la sustanciación de derechos desde una perspectiva de género y diversidad sexual y corporal. Como correlato de estas transformaciones, se expone la potencia del cambio social con respecto a las tensiones y las resistencias al binarismo sexo-genérico que impregnan el sistema educativo y se evidencian las maneras en que se impulsaron cambios epistémicos y epistemológicos que construyen nuevos horizontes políticos en las prácticas pedagógicas.

Palabras clave: Educación sexual integral - movimiento pedagógico binarismo sexo-genérico - diversidad

\section{Abstract}

Since the enactment of a national law in 2006, Comprehensive Sex Education (ESI) has been institutionalized as a public policy in Argentina. Various cultural struggles permeated this process and were redefining the epistemic bases of this educational policy. In this work, from a critical ethnographic perspective, we show how a pedagogical movement was produced around this process of political construction, involving social movements, political activisms and state organisms, which, in conjunction with legislative changes that implied democratization of citizenship, promoted the substantiation of rights from a perspective of gender and sexual and corporal diversity. As a correlate of these transformations, the power of social change is exposed with respect to the tensions and resistances to sex-gender binarism that pervade the educational system and the ways in which epistemic and epistemological changes were promoted to build new political horizons in pedagogical practices.

Keywords: Comprehensive Sex Education - Pedagogical Movement Sex-Gender Binarism - Diversity 


\section{Introducción}

La Educación Sexual Integral (ESI) ${ }^{2}$ constituye un escenario institucional clave de diversas luchas culturales en Argentina. Su institucionalización, a partir de 2006, estuvo atravesada por la intervención de diferentes colectivxs políticxs que encontraron la ocasión para reorientar los debates sobre los contenidos y los enfoques de esta política pública. Al mismo tiempo, estas intervenciones, principalmente desde lxs colectivxs de los feminismos, los movimientos de mujeres, las disidencias y las diversidades sexo-genéricas, fueron logrando una legitimidad pública cada vez mayor para ampliar y transformar las bases epistémicas de la ESI con respecto a las coordenadas sexo-genéricas de la ciudadanía. Estas diferentes gestiones intersectoriales fueron tramando los sentidos locales de la política con ciertos devenires imprevistos en relación con su formulación inicial. Desde sus comienzos, cada vez con más fuerza, la ESI se fue (re)organizando a través de estos compromisos políticos para mediar y transformar la vida emocional y afectiva. Un objetivo central de estas diferentes perspectivas de intervención política y pedagógica en el campo de la ESI ha consistido en estimular y garantizar una convivencia más democrática y justa en la tan compleja supervivencia en el capitalismo, actualmente estallado por la pandemia del COVID-19.

Nuestro aporte en este dossier retoma y profundiza un trabajo anterior (Lavigne y Péchin, 2021) en el que elaboramos una cartografía crítica de las profundas transformaciones epistémicas en el enclave institucional de la ESI. Allí, etnografiamos cómo se produjo un movimiento pedagógico en torno a la ESI, que no puede reducirse a la institucionalización directa a partir de la puesta en marcha de la Ley Nacional No26.150 de ESI para Argentina, sino que integró diferentes movimientos sociales que hicieron de una ley -con no pocos defectos (Barrancos, 2009) - una plataforma pedagógica muy fuerte, que se fue consolidando y potenciando desde el momento de su sanción (2006) e, incluso, desde las demandas y disputas previas y, también, desde las que se fueron suscitando a partir de su implementación. Procuramos dar cuenta de ciertos cambios en las estructuras de conceptualización a la vez que éstas van modificando las prácticas de enseñanza y aprendizaje en una dialéctica incesante, que tiene una aceleración muy difícil de describir por su ubicuidad, su intensidad y su progresión. Proponemos, así, un mapeo no exhaustivo de estas transformaciones en diferentes enclaves institucionales que nos permite mostrar este movimiento $y$, al mismo tiempo, reconocer ciertas genealogías de su situación histórica.

Desde la intersección de dos etnografías realizadas en el marco de dos

2 La ley 26.150, sancionada en 2006, crea el Programa Nacional de Educación Sexual Integral, al mismo tiempo que impulsa una transversalidad entre todas las áreas disciplinares y en cada nivel educativo para priorizar un enfoque pedagógico con perspectiva de género, derecho y diversidad que reorganice las prácticas áulicas, los modos de reflexión implicados en ellas y los saberes y los conocimientos puestos en juego. En la CABA, se sanciona una ley propia, №2110, también en el mes de octubre de 2006, mientras que otras jurisdicciones crearon también sus leyes específicas, adhirieron a la Ley Nacional o crearon resoluciones ministeriales en torno a la ESI. 
investigaciones doctorales (Lavigne, 2016 y Péchin, 2012), que fueron desarrolladas durante el proceso de construcción política de la ESI en tanto política pública, y nuestras respectivas posteriores actividades investigativas, docentes y activistas, nos proponemos trazar un abordaje multisituado (Marcus, 1995) sobre diferentes agencias del estado, actorías y jurisdicciones del país. El propósito es historizar críticamente y visibilizar etnográficamente las agencias activistas, políticas e institucionalizantes que participan en la producción de la ESI como dispositivo para avanzar en una sustantivación concreta de derechos desde una perspectiva de género y de diversidad. Mostramos prácticas institucionales, pedagógicas, docentes y estudiantiles, a la vez que referencias investigativas, bibliográficas y pedagógicas nodales de esta construcción en Argentina.

La perspectiva crítica del abordaje cualitativo nos permite producir metodológicamente este texto a través de la técnica del bricollage etnográfico, exponiendo una constelación de imágenes a partir de una heterogeneidad empírica de lo multisituado del análisis etnográfico: esas múltiples referencias componen el mosaico del bricollage que muestra un movimiento político-pedagógico en ciernes. La perspectiva etnográfica y crítica del trabajo (Rockwell, 2009; Noblit et al., 2004) que, a su vez, va trazando un juego arqueológico con los recursos que se fueron elaborando para materializar esta construcción política (bibliografía, cursos y programas, materiales didácticos, etc.), nos permite mostrar cómo la creciente intensificación de las políticas feministas, trans, travestis, no-binarixs y queer/cuir reorganizan las fronteras hermenéuticas del binarismo sexo-genérico para la implementación de la ESI como política pública. Historizamos, así, este proceso en vínculo con algunos cambios legislativos que implicaron una democratización de la ciudadanía a la vez que fueron puntos concretos de inflexión para la implementación de la política pública en sí.

\section{Configuración política de la ESI como movimiento pedagógico: activismos que construyen saberes}

La ESI se fue conformando y consolidando como un movimiento pedagógico (Colectivo Mariposas Mirabal, 2019) en sí mismo que articuló movimientos feministas y de las disidencias sexo-genéricas, a la vez que sindicatos, organizaciones sociales y otros ámbitos institucionales. En este proceso, docentes, otrxs actorxs de la escolaridad y del campo pedagógico -como equipos directivos, tutorxs, equipos de orientación escolar, gabinetes psicopedagógicos, entre otros- y otras agencias del estado -como defensorías y centros de salud- se vieron interpeladxs por esta oportunidad histórica para producir esta intervención institucional. La ESI se fue volviendo, así, un compromiso político para una legión cada vez más creciente de docentes, formadorxs y capacitadorxs docentes, que lo fuimos asumiendo como un activismo en sí mismo, como ocasión política para transformar las condiciones 
institucionales de una regulación cultural clave de la vida humana. Se fueron armando estrategias individuales y colectivas entre colegas docentes y no docentes, al mismo tiempo que estudiantes que se comprometieron con convicción política con las luchas emancipatorias principalmente feministas y TLGBQI++3 que guían el dispositivo de la ESI.

Estas diferentes prácticas que problematizamos nos permiten mostrar una proliferación de sentidos de la ESI, así como profundas transformaciones epistémicas que expresan la enorme fuerza y potencia de este movimiento pedagógico. Desde los márgenes, distintos modos de activismos han ido ganando legitimidad para imponerse en los espacios de producción, circulación y pedagogización de los conocimientos para torcer esas claves sexo-genéricas conservadoras de inteligibilidad de lo humano, propiciando la descriminalización y la despatologización (Suess, 2014). Las actorías de este movimiento son sumamente heterogéneas, con diversos propósitos y modalidades de intervención. Están constituidas por organizaciones estudiantiles, de familiares y otras intervenciones disciplinares y activistas en el ámbito pedagógico. Fueron elaborando herramientas y estrategias de trabajo que tensionaron los modos más convencionales desde los márgenes, más allá de los ámbitos formales tradicionales de construcción política en el campo educativo, por ejemplo, las asociaciones gremiales docentes. En el campo escolar fueron asumiendo especial notoriedad diferentes organizaciones juveniles. En el marco de la población docente, se conformaron "frentes" o "redes" con énfasis y ejes diferentes, pero con el propósito en común de impulsar la implementación de la ESI desde la construcción colectiva. ${ }^{4}$

\footnotetext{
3 Usualmente suele presentarse el acrónimo para nuclear las posiciones trans, travestis, lésbicas, gays, bisexuales, queer/cuir, intersex y el signo más que permite incluir otras, bajo el ordenamiento LGBT o LGBTQI+. En los años previos a la formación de la FALGBT (Federación Argentina LGBT) en 2006, a partir de las reuniones federales llevadas a cabo en la ciudad de Rosario por la mayor parte de las organizaciones TLGBQI+ de Argentina, se visibilizó el reclamo de las organizaciones lésbicas para modificar la centralidad de la hegemonía gay masculina del movimiento que se expresaba habitualmente en el acrónimo GLBT. A su vez, catalizado por el impulso de los movimientos feministas, se fue corriendo el uso hacia LGBTQI y, más recientemente, se le agregó también el "+" como estrategia inclusiva de otras posiciones identitarias y políticas. En los últimos años, dentro del movimiento se fueron escuchando los reclamos de las organizaciones trans y travestis que denuncian la centralidad de la hegemonía cis-género. Se impulsó entonces el uso TLGBQl+ para redefinir el lugar político de lxs colectivxs trans y travestis desde algunas agrupaciones, entre las que ha sido pionera OTRANS de la ciudad de La Plata, Provincia de Buenos Aires. Si bien suele subsumirse el posicionamiento identitario no binarie bajo las luchas trans y/o queer/cuir, sin embargo, cada vez cobra mayor fuerza como colectivx, discutiendo también su visibilización en el acrónimo que nombra al movimiento de las disidencias y/o las diversidades sexo-genéricas. En este escrito, en el que historizamos algunas inflexiones impulsadas por los activismos políticos con respecto a los abordajes pedagógicos sobre las diversidades y las disidencias sexo-genéricas en la escolaridad y en otros ámbitos institucionales, hemos optado por hacernos eco de este corrimiento para enfatizar los procesos de construcción política y las transformaciones epistémicas y hermenéuticas.
}

4 Entre otras organizaciones, encontramos la Red de docentes por el Derecho al Aborto Legal, Seguro y Gratuito de la Campaña Nacional por el Derecho al Aborto Legal, Seguro y Gratuito, que tiene sus antecedentes en 2014 en la Ciudad de Buenos Aires y se constituye en una red federal con el correr de los años. Tiene un propósito central en cuanto a la ESI que consiste en trabajar el derecho al aborto en las escuelas. El Frente Popular por la ESI (integrado por docentes, sindicatos, estudiantes, organizaciones sociales, comunicadorxs e intelectuales) se conformó durante la gestión del gobierno macrista, en el año 2018, en "defensa" de la ESI y para denunciar y contrarrestar la política de ajuste y falta de presupuesto para garantizar la ESI a nivel nacional. La Red Nacional de Docentes Trans propone una pedagogía trans e impulsa la ESI como oportunidad para, desde las escuelas, permitir otros existenciarios que no reproduzcan las vivencias habituales de discriminación y expulsión del sistema educativo, generando un espacio de reflexiones colectivas para la acción entre docentes trans, fundamentalmente, de diferentes jurisdicciones. 
A través del movimiento pedagógico que se fue gestando en torno a la ESI, se fue construyendo cada vez mayor legitimación para otros saberes hasta entonces no reconocidos, así como para otras formas de producción de conocimientos de diversas procedencias (campo popular, activismos feministas, cuir/queer, trans y travestis, entre otras), que interpelan el propio campo pedagógico. A partir de las articulaciones mencionadas, se fueron produciendo tanto prácticas y experiencias como publicaciones, recursos y materiales con circulaciones que proliferaron sus alcances a través de capacitaciones, encuentros, foros, jornadas, locales y nacionales, entre otros formatos, con el propósito de discutir y producir saberes y sentidos críticos en torno a la ESI.

Un conjunto de publicaciones sistematizó y socializó la producción de nuevos saberes. Se trata de conocimientos que discuten fuertemente las tradiciones, incluso desde la construcción de otras perspectivas desde los espacios académicos que, a su vez, invierten la legitimidad unívoca que venía teniendo este ámbito en relación con el resto de los espacios para arrogarse una hegemonía en la producción de saberes. Una serie de materiales pedagógicos y producciones provenientes de diferentes ámbitos y movimientos sociales fueron incorporados en las tramas del saber escolar por parte de una relevante cantidad de docentes. Resulta imposible reponer aquí un exhaustivo estado de la cuestión en cuanto a dar cuenta de todos los aportes producidos desde organizaciones, instituciones y activismos más independientes y su enorme proliferación. Algunos de ellos han tenido especial significación en el contexto escolar, ya sea como materiales didácticos, como literatura citada en los programas o como mapeo teórico, conceptual y político de las prácticas encarnadas por docentes y el propio estudiantado.

En nuestro país, desde la década del noventa y con inmenso empuje y proliferación en los contextos académicos, se conformaron equipos de investigación y producción escrita, consolidando diálogos entre las esferas de la gestión educativa, la formación docente y las universidades. ${ }^{5}$ En esta línea, diferentes equipos de investigación y

\footnotetext{
5 Aquí cabe referir a una vasta producción. Mencionamos apenas algunos equipos de diferentes jurisdicciones, academias y organizaciones del país que revisaron las maneras en que el sistema educativo está constitutivamente signado por el sistema sexo-género, incluso más allá de una expresa política educativa como la que aquí enfocamos centralmente. El equipo dirigido por Graciela Morgade, cuyas investigaciones tienen una larga historia de estudios en el campo de la educación sexual con perspectiva de género en la FFyL de la UBA enfatizó que "toda educación es sexual" (2011) y produjo una colección denominada La Lupa de la ESI. Investigadorxs del Instituto de Investigaciones en Estudios de Género (IIEGe) de la FFyL publicaron Género y sexualidades en las tramas del saber. Revisiones y propuestas (2009), coordinado por Elizalde, Felitti y Queirolo. Lxs autores procuran una revisión crítica desde diferentes disciplinas (Historia, Lengua y Literatura, Artes, Comunicación) y propuestas concretas de trabajo. Desde el IIEGe se desarrollaron diversas investigaciones y escritos que aportaron al campo de educación, géneros y sexualidades. El Programa de Estudios Queer (FFyL) constituyó "un espacio de articulación de proyectos de docencia e investigación con las prácticas de activismo y militancia en movimientos políticos contra la represión y la discriminación por géneros, orientación sexual e identidad de géneros". En Cipolletti (Río Negro) se conformó el programa de posgrado de Especialización en Educación y Estudios Interculturales, géneros y sexualidades en la Facultad de Ciencias de la Educación de la Universidad Nacional del Comahue, dirigido por Graciela Alonso y Raúl Díaz. Desde Neuquén y con amplia articulación en toda la Patagonia, principalmente vinculando equipos de trabajo en los institutos de formación docente y las universidades nacionales (Neuquén, Cipolletti, El Bolsón, San Martín de los Andes, Esquel, Junín de los Andes, Bariloche, Trelew, Puerto Madryn, Epuyén, entre otros) desde principios del siglo XXI, la colectiva feminista "La Revuelta" entrelazó intensamente
} 
producción de recursos actualizaron los alcances de los contenidos definidos en el marco de esta política educativa y contribuyeron con la edición de publicaciones. Recalcamos, entonces, que los activismos fueron impactando al mismo tiempo una autoridad hermenéutica, una autoridad pedagógica y una autoridad interpretativa. Y como contenido y correlato de esa interpelación, se logró una fuerte transformación epistémica y un giro epistemológico que implicaron dar visibilidad y buscar formas adecuadas para denominar las corporalidades y las identidades sexo-genéricas que, históricamente, fueron clausuradas por las ritualidades sexo-genéricas que establecen los binarismos macho/hembra, varón/mujer y masculino/femenino como correspondencias naturalizadas e ineludibles.

Especialmente, en los últimos años, se instaló una reflexión pública sobre la experiencia trans y travesti en la infancia y la adolescencia en el contexto educativo (Báez, 2013), además de la participación adulta de personas trans y travestis como docentes, formadorxs y capacitadorxs docentes y estudiantes, a la vez que otras experiencias identitarias no binarixs que redefinen los modos de habitarlas, o bien proponen otras narrativas identitarias o contra-identitarias. Estas, además de las perspectivas trans, transgénero y travesti, también recuperan la perspectiva política queer y su reterritorialización sudaca como cuir, a la vez que las críticas a lo queer/ cuir y los feminismos.

\section{Formación y capacitación (entre) docentes como escenarios claves para las transformaciones epistémicas}

En diversos dispositivos de capacitación, resultaron claves las articulaciones con activistas de diferentes organizaciones del campo de los feminismos como de las disidencias sexo-genéricas, la incorporación de producciones visuales, escritas y audiovisuales que permitieran incorporar en la labor pedagógica esas transformaciones epistémicas que venían siendo traccionadas. ${ }^{6}$ Los espacios en el

\footnotetext{
La militancia con la labor académica desde trayectorias docentes, investigativas y activistas. Elaboraron materiales pedagógicos como: "La Revuelta Pedagógica", "No se nace heterosexual" y mantienen una producción digital activa al respecto. Por su parte, la maestra, escritora y activista val flores creó un Blog denominado "Escritos heréticos" donde se compilan diferentes producciones feministas, queer, cuir. En la Universidad Nacional de Córdoba se radicaron otras investigaciones y el programa de doctorado en estudios de género. Más recientemente se conformó el Centro Interdisciplinario de Estudios y Políticas de Género (CIEPoG) en la UNTREF (Prov. Bs. As.). El Centro Cultural Tierra Violeta desde 2012 constituye un espacio de acción y producción colectiva feminista en la CABA. En cuanto a espacios de formación docente podemos mencionar: el equipo de ESI de la Escuela de Maestros (ex CePA, Ministerio de Educación de la CABA); el Postítulo de Especialización del J. V. González; la Diplomatura en ESI (FFyL-UBA) y el Ārea: Educación, género y sexualidades de la Carrera de Especialización en Educación: Pedagogías para la igualdad en contextos socioeducativos diversos (FFyL-UBA); y la conformación de la Diplomatura en Educación, Género y Sexualidades (UNICEN, Tandil).
}

6 Entre estas producciones relevamos a "Mujeres públicas", como colectiva feminista desde el campo del arte de la ciudad porteña, cuyas producciones fueron apropiadas como recurso pedagógico para discutir los mandatos y desigualdades de género y la heternormatividad. La artista sudaca trans, Susy Shock, publica "Crianzas. Historias para crecer en toda la diversidad" (2016), que rápidamente comenzó a circular en los espacios de formación docente y en las propias escuelas, porque habilita a valorar la diversidad de la sexua- 
ámbito público y privado para la formación y la capacitación de docentes en materia de ESI fueron cada vez más atravesados por una proliferación significativa de activismos feministas, TLGBQI+, de derechos humanos y de protección integral de derechos de niñxs y adolescentes. Gran parte de las docencias, las formaciones y las capacitaciones docentes está sostenida por activistas, a la vez que gran parte de los activismos se volcaron a intervenir de diferentes maneras en la escena educativa y, específicamente, en la escolar. Así, impactaron las lógicas más tradicionales del binarismo cis-hetero-patriarcal en la cultura escolar y los encuadres educativos en general.

Durante los últimos años, los espacios de capacitación docente de diversos niveles educativos, incluyendo el nivel superior y las universidades, fueron escenarios claves en los que se produjeron encuentros abocados a cierta sensibilización pedagógica para alojar y visibilizar institucionalmente a las personas TLGBQI+ que forman parte del sistema educativo. El reconocimiento de esta realidad significa adecuar los abordajes de la ESI para incluirlas en las prácticas pedagógicas, principalmente en las discursivas. Algunos enfoques fueron radicalizando la perspectiva $y$, en lugar de trabajar una propuesta de inclusión, en cambio, también fueron poniendo en reflexión, además de la invisibilización y la exclusión TLGBQI+, la obligatoriedad cis-heterosexual que asumen las narrativas institucionales públicas y privadas en su conjunto. ${ }^{7}$ Trabajar con y desde las diversidades y las disidencias sexo-genéricas en las escuelas implica un desafío político, social y cultural que interpela, fundamentalmente, tanto el núcleo de debates de la ESI como los propios insumos conceptuales que los delimitan en la formación y la capacitación docente, en particular, como también en los otros espacios académicos como las universidades y otros centros de investigación y docencia articulados con ellas para la producción, circulación y legitimación pública de los conocimientos y los saberes.

Con una convocatoria cada vez más creciente -con drásticos aumentos de la matrícula de cada instancia ofertada y la creación de largas listas de espera y personas que quedaron sin vacante en sucesivas cohortes-, también se multiplicaron los ámbitos de formación y capacitación en los distintos espacios de la educación formal, no formal y otros ámbitos institucionales y de organizaciones sociales no

Lidad humana y al mismo tiempo, que las voces de personas trans sean reconocidas como forma de compartir saberes y conocimientos. Anteriormente, Gabriela Mansilla publicó Yo nena, yo princesa, armado a partir de su diario íntimo como mamá de una niña trans que logró hacer su escolaridad con un DNI que refleja su identidad autopercibida a partir de la lucha incesante de su madre que se volvió una referente central en los debates educativos e impulsó la conformación de la campaña Infancias trans sin violencia ni discriminación, que tiene ya un alcance global. Es necesario destacar que Luana fue la primera niña trans del mundo que logró hacer su escolaridad con su verdadera identidad, la que siente y reclamó para sí misma.

7 Estas últimas narrativas contienen un congruente régimen de: parejas monogámicas, jerarquización de lo masculino sobre lo femenino, sobrevaloración del macho como paroxismo de la supremacía del varón y del género masculino, inferiorización y subordinación de la mujer y de lo femenino, subestimación de toda hibridación en la identificación y la expresión personales de género y de toda orientación y toda práctica sexuales que no busquen normalizarse según el régimen binario cis-hetero-patriarcal de inscripción genital de los cuerpos desde el nacimiento que produce la idea de una cierta autenticidad de origen sobre la que se organiza la experiencia sexual, genital y de género de la persona como eje constitutivo de cualquier otra experiencia personal y vincular, individual o colectiva. 
directamente vinculados con la educación en sí. Esto se dio en el marco de las diferentes jurisdicciones y con cierta especificidad de implementación en cada región, pero también en una intersección más amplia con los debates sobre cuerpxs ${ }^{8}$, géneros y sexualidades desde distintos enfoques y campos de estudios tendientes a la interdisciplinariedad.

Algunas experiencias del campo popular movilizaron espacios pedagógicos alternativos al sistema educativo formal, para habilitar pedagogías críticas que aportaron fuertemente en la construcción de herramientas y experiencias de ESI. El Bachillerato Popular Travesti Trans Mocha Celis en la CABA fue pionero y también en otras jurisdicciones se fueron abriendo espacios semejantes, como el Centro Educativo Trans de Puertas Abiertas en la provincia de Tucumán. En este sentido, varios otros bachilleratos populares y otros encuadres populares de educación y escolarización se fueron encuadrando en una implementación de la ESI con perspectiva de derecho, género y diversidad.

El enfoque de la interseccionalidad también compuso otras coordenadas para reconocer las diferentes variables que se intersectan en la configuración identitaria de personas como colectivxs, al considerar el cruce entre género, orientación sexual, etnia, discapacidad, clase social y/o edad. Al permitir analizar cómo se solapan diferentes formas de discriminación, convocó a contemplar la diversidad desde múltiples dimensiones. Así, el activismo gordx, las discapacidades, la diversidad funcional y la diversidad corporal fueron experiencias cada vez más visibilizadas desde los activismos que así lo impulsaron, articulando sus prácticas y la institucionalización de la ESI.

Los cambios en los marcos normativos propiciaron ciertos énfasis y fueron aprovechados como instancias para producir sinergias para ampliar, diversificar y procurar nuevos enfoques, conceptualizaciones de géneros, sexualidades y derechos, en tanto herramientas concretas para el trabajo en las escuelas y otros ámbitos educativos.

\section{Conquistas legislativas para una ampliación democrática de la ciudadanía y reorientaciones epistémicas de la ESI}

La conquista legislativa de derechos arrebatados a través de décadas de activismos feministas y TLGBQI+ se va sustanciando como logro institucional y cambio cultural concretos en tanto se democratizan nuestras posibilidades discursivas: mucho más que para meramente clasificar nichos subjetivos con necesidades especiales, en

8 Las prácticas feministas, queer-cuir, trans y no binarixs habitualmente intervienen la palabra "cuerpo" para señalar el paroxismo cis-hetero-patriarcal de un androcentrismo que, si bien no está produciendo una declinación directamente sobre lxs sujetxs, con las alternativas "cuerpx" o "cuerpa", recuperan un gesto de la interrumpción de género propio del lenguaje inclusivo o recurren a una declinación femenina para denunciar el patrón masculino que, como universal, condiciona las formas de construir y vivir las corporalidades. 
cambio, para que lxs sujetxs deseantes que transitamos las instituciones tengamos también lugar como sujetxs deseadxs.

El movimiento pedagógico que constituye la ESI está en consonancia con la "marea verde"9 y el avance institucional general en materia de ampliación de la ciudadanía para garantizar los derechos a la identidad de género $0^{10}$ y a los vínculos familiares con independencia del sexo de los cónyuges, así como otros marcos normativos que traccionaron transformaciones en las formas de regulación de los géneros y las sexualidades y consolidaron políticas que, en gran medida, fueron demandadas durante décadas por parte de los diversos activismos intensificados y resignificados en la última década.

Así como podemos referir el año 2015 como momento en que se tornó colectivamente inaceptable la cantidad de femicidios, en tanto crímenes de odio, y se produjo la masiva movilización que instaló la conceptualización acuñada por la organización "Ni una menos" como su nombre y con alcance transnacional, también en el marco de la política educativa durante aquel fin de año se decidió instalar una jornada específica para abordar en las escuelas las violencias de género a partir de la Ley $\mathrm{N}^{0} 27.234 ; 11$ es indispensable mostrar las masivas demandas por procurar la soberanía sobre lxs propixs cuerpxs que se sostuvieron en la formulación del proyecto de ley para garantizar el acceso libre y gratuito al aborto en 2018, hasta obtener la sanción de la Ley No 27610 de Acceso a la Interrupción Voluntaria del Embarazo en 2020. En esa batalla, se involucraron múltiples actorías del movimiento pedagógico que hicieron de los espacios públicos y los ámbitos escolares territorios fundamentales de visibilización de demandas, movilización, debate y construcción de argumentos para sostener la disputa socialmente instalada por doquier.

Estos procesos que conllevan estas fisuras y transformaciones epistémicas deben ser comprendidos en contextos históricos concretos, producto de disputas y construcciones de sentido social que no están exentas de su anulación y pueden retrotraerse. En esa tensión, queremos instalar la advertencia respecto de los permanentes enclaves de disputa que incluyen voces desde el disenso y la oposición flagrante al ejercicio de ciertos derechos alcanzados por las personas, sobre todo TLGBQI+, relevadas a través de prácticas y expresiones de odio, rechazo y discriminación. ${ }^{12}$

9 "Marea" en referencia a la masiva movilización de diferentes colectivas feministas y de las disidencias sexo-genéricas que luchan por el derecho al aborto, y "verde" en alusión al color adoptado por la Campaña Nacional por el Derecho al Aborto Legal Seguro y Gratuito.

10 Ley N²6.743 de Identidad de género, sancionada el 9/5/2012.

11 Ley N027.234 Educar en Igualdad: Prevención y Erradicación de la Violencia de Género, sancionada el 26/11/2015.

12 Los grupos conservadores, fundamentalistas y autoritarios frente a las políticas sobre sexualidades en Latinoamérica y en nuestro país en particular se encuentran interviniendo activamente en los sistemas de derechos humanos nacionales e internacionales. En nuestro país la organización transnacional denominada "Con mis hijos no te metas" se opone tajantemente a lo que denominan la "ideología de género" e impulsó campañas contra la implementación de la ESI. 
Diversos plexos normativos complementan los ya mencionados hasta aquí y se articulan para garantizar derechos históricamente reclamados al estado desde una nueva matriz institucional que van formulando las políticas públicas impulsadas en estos marcos legales y promoviendo una ampliación concreta de nuestra democracia. ${ }^{13}$ Este conjunto de normativas mencionadas -que enmarcan y potencian los derechos conquistados- impactaron fuertemente sobre los cimientos epistémicos de la ESI y permitieron legitimar y desplegar otras prácticas en la escolaridad. Sin embargo, la gestión de las mismas no es lineal y técnica, sino que requiere de procesos políticos para reorientar la formación, la capacitación y la sensibilización para la revisión de las propias prácticas y los presupuestos en los que se basan. Para el abordaje de una perspectiva de género que reflexione sobre la presunción automatizada del binarismo en el campo educativo y las desigualdades que supone esta construcción, resultó indispensable procurar espacios de sensibilización y capacitación ${ }^{14}$ en pos de transformar críticamente nuestras prácticas institucionales. ${ }^{15}$ Estas trayectorias se sitúan sobre ciertas narrativas que ya habían comenzado a fisurar los sentidos hegemónicos de la estabilidad sexo-genérica de un hetero-patriarcado en cuestión y rediseñan, así, los sentidos epistemológicos de la construcción de nuevas formulaciones a partir del profundo cambio cultural ocasionado por los debates mediáticos y sociales instalados en el marco del debate legislativo y la sanción de la ley del llamado "matrimonio igualitario", que permitió la ampliación del contrato matrimonial para toda pareja con independencia del sexo de las personas que la conforman. Además, contribuyó con la visibilización de una vasta diversidad vincular -que incluso no se agota con las posibilidades de la pareja monogámica- a la vez que con la profunda reformulación de la concepción de las formas legítimas de familias.

En las escuelas, esto supuso una redefinición de los relatos disponibles para albergar esta diversidad de configuraciones familiares que, aunque siempre existieron, ahora se sirven de un contrato legitimado por el Estado en la figura del llamado "matrimonio". 16 En tanto que las actualizaciones de los documentos oficiales

\footnotetext{
13 Ley N²6.485/09 de protección integral para prevenir, sancionar y erradicar la violencia contra las mujeres en los ámbitos en que desarrollen sus relaciones interpersonales; Ley №26.618/10 de matrimonio igualitario; Ley $N^{0} 26.657 / 10$ de salud mental; Ley $\mathrm{N}^{\circ} 26.743 / 12$ de identidad de género; Ley $\mathrm{N}^{\circ} 26.791 / 12$ de homicidios agravados por razones de género: femicidios y crímenes de odio; Ley N²6.862/13 de Reproducción médicamente asistida; Reforma del Código civil y comercial Ley №26994/15; Ley N²7.234 Educar en Igualdad, Prevención y Erradicación de la Violencia de Género; Ley Micaela N²7.499/18 de Capacitación obligatoria en genero para todas las personas que integran los tres poderes del estado; Ley №27.610 Acceso a la Interrupción Voluntaria del Embarazo.
}

14 Estos espacios fueron conformados de modo dispar y con diferentes enfoques en las ofertas de los centros de formación y capacitación docente de cada jurisdicción.

15 En consonancia con esta necesidad, en el año 2019 se estipula una expresa política pública que supone una "Capacitación obligatoria en género y violencia de género para todas las personas que se desempeñan en la función pública, en los poderes Ejecutivo, Legislativo y Judicial de la Nación" (Ley Nacional º27499). La denominada Ley Micaela remite a un asunto específico de ESI ganado por Los activismos: una exigencia pedagógica para con el Estado en su dimensión humana e intersubjetiva.

16 Un año después de sancionada la Ley, la organización Les Madres, integrada por familias de lesbianas madres y futuras madres, 
y materiales didácticos conllevan tiempos específicos de producción y reelaboración, las instituciones educativas gestionaron la selección de materiales con criterios que procuraban una heterogeneidad de maneras de visibilizar y nombrar esa diversidad. En miras de esta necesidad, se fueron creando diferentes recurseros para circular la compilación de bibliografía sugerida con diferentes alcances y sistematizaciones, secciones específicas en las librerías e incluso algunas especializadas.

Estas diversas leyes, que fueron el producto de años de disputas políticas para la incorporación de garantías concretas para derechos históricamente no reconocidos, cada una de ellas a su modo, trazaron sentidos que estimularon este giro epistémico/ epistemológico al que aquí nos referimos. Estos avances legislativos y las políticas públicas afines fueron articulándose entre sí y haciendo máquina con las resignificaciones culturales de las identidades y de las diferencias que quedaron al margen de las segmentaciones sociales que, históricamente, reconoce el basamento binario del derecho. Más allá de los logros de jurisprudencia y fallos a favor de cierta neutralidad de género en los casos concretos en los que algunxs ciudadanxs desafiaron su encasillamiento binario, incluso con el avance legal e institucional que significó la Ley de Identidad de Género (2012) a partir de la que las personas podemos optar autónomamente por quedarnos en la rúbrica sexo-genérica que nos fuera impuesta o cambiar nuestro registro civil del propio sexo y nombre, el nacimiento y/o la modificación posterior siguen signados por una ineludible inclusión en los exhaustivos casilleros binarios de M o F. ${ }^{17}$

Esta legislación argentina sobre la identidad de género garantiza, por primera vez a nivel mundial, el reconocimiento estatal de la autopercepción de la persona sin diagnosis psico-médica ni judicialización, sólo a través de un trámite administrativo simple. A su vez, ya el hecho mismo de (re)habitar y (re)transitar aquel binario desde otro recorrido y con otra construcción que una asimilación del lugar asignado en el nacimiento, reubica la identidad femenina o masculina en un sentido que también lo desafía, mostrando los matices intermedios que se vivencian entre la obligatoriedad polar de asumirse como un caso de lo masculino o de lo femenino, excluyentemente. Aquí se inscriben también algunas narrativas identitarias no binarixs, tensionando a

elaboró un recurso llamado "Familias comaternales. Guía para personal educativo" que contó con Declaración de Interés Educativo de parte del Ministerio de Educación de la Nación Argentina (mayo de 2011).

17 Es importante destacar que, en noviembre de 2020, se produce la campaña Todes con DNI, que es impulsada por un grupo de personas no-binaries que necesitan cambiar sus documentos para que se ajusten a sus identidades de género conforme con la Ley de Identidad de Género sancionada en 2012. En el texto de la campaña se explica que "Desde fines de 2018 los Registros Civiles de distintas provincias rectificaron muchas partidas de nacimiento indicando un género diferente a masculino/femenino o no indicando nada en ese campo. Muchos de esos casos no salieron a la luz. Hasta el día de hoy, ninguna de esas personas obtuvo su DNI. Tampoco pueden tramitar su pasaporte. Paralelamente, muchos Registros Civiles en todo el país se están negando a tomar pedidos de rectificación de partidas de nacimiento. Para muches, sus documentos no dicen ni su nombre ni su género elegidos, no reflejan quiénes son". Esto es particularmente relevante dado que muchxs estudiantes manifiestan así su identidad autopercibida. Esta situación vulnera a su vez el artículo 12 de la ley que exige un trato digno conforme al género y al nombre elegidos por la persona según su autopercepción, aunque no hagan un cambio registral, pero otorgándole el derecho a hacerlo. 
veces la identidad registral y la propia expresión de género.

Se impusieron profundos cambios en las conceptualizaciones y enfoques sobre cuerpxs, géneros y sexualidades, así como adaptaciones curriculares y capacitaciones del personal educativo con el fin de incorporar las implicancias de estas políticas en el contexto de la vida escolar y, también, al mismo tiempo avanzar a partir de las bases que establecen estos marcos normativos como envión para un cambio mucho más profundo. Esta ley, por lo tanto, radicaliza el modo en el que la ESI tiene que repensar esa educación ciudadana, en la medida en que ser varón o mujer ya no es patrimonio exclusivo de la asignación que produzca el estado a través de equipos médicos, tutorxs legalxs y juzgados. La Ley de Identidad de Género incorpora, así, la autonomía y el respeto por la vivencia interna y la expresión de niñxs y adolescentes, exponiendo su concepción en tanto sujetxs de derechos y, más aún, de sujetxs políticxs y sujetxs deseantes.

Esto implica propiciar cambios en las regulaciones internas y los instrumentos administrativos que procuren el cambio de nombre de acuerdo con la simple mención del estudiantado, del cuerpo docente o no docente. De esta manera, se produce un quiebre fundamental en tanto que el estatuto de legitimación pública de la identidad de género se corre de la perspectiva biológica, psico-médica y judicial para garantizar la consideración de la vivencia interna del género tal como cada persona la siente, siendo el respeto inalienable de la autonomía y de la vivencia personal el vector prioritario por sobre la asignación y la determinación de lxs otrxs. Se avanza, entonces, hacia un corrimiento epistemológico desde un foco puesto en la articulación clínico-pedagógica de la patologización hacia un empoderamiento político-pedagógico de estrategias para el reconocimiento de la autonomía personal para la propia definición de la identidad de género como derecho inalienable de la persona, cualquiera sea su edad y de acuerdo a cómo lo manifiesta, expresa y comunica según sus capacidades.

\section{Fisuras al binarismo y problematización pedagógica de la invisibilización/ exclusión trans, travesti y no binarie en la escolaridad}

El enclave educativo ha sido especialmente fértil para propiciar una reformulación profunda y alternativa al binarismo sexo-genérico impregnado en el lenguaje y en la lengua, que se instituye como axioma fundacional de la circularidad conceptual y explicativa entre los cánones estéticos y los estatutos éticos de los cuerpos y sus gestualidades en su circulación individual por los espacios públicos. Desde aquella transformación política en la que el Estado Argentino formaliza una definición específica de la identidad de género, diferentes prácticas del sistema educativo formal entraron en conflicto en relación con las formas de intervención, la organización de la vida institucional y los propios recursos pedagógicos, e incluso 
administrativos, disponibles. Tres escenas permiten mostrar cómo se fueron tejiendo esas experiencias que fisuraron los modos de inteligibilidad binaria y cómo fueron tensionadas la manera de gestionar el uso de los espacios, las áreas curriculares y los recursos didácticos.

En cuanto a la configuración y organización de los espacios como los baños en las escuelas, se dieron innumerables conflictos cuando los habitan personas trans y no binarias (como estudiantes, docentes y no docentes), al formularse desde conducciones, equipos docentes y estudiantes el interrogante respecto a qué baño le/s corresponde, bajo la habitual clasificación binaria: mujeres o varones. En algunas escuelas se habilitaron baños no binarixs o mixtos o unisex, en algunas ocasiones denominados "multigénero". Se fue modificando, así, la distribución sexualizada tradicional en la búsqueda de modos más inclusivos con respecto a la diversidad sexo-genérica y corporal que también habita la escolaridad.

La educación física como una de las tradicionales áreas curriculares fue un ámbito que generó fuertes tensiones al momento de garantizar el derecho a la educación de estudiantes y docentes trans y no binaries, mientras en diferentes oportunidades relevadas se les impidió participar en la clase dividida entre varones y mujeres, de acuerdo con la identidad de género cuando ésta no era coincidente con el registro escolar o el DNI de las personas. También, se tornó un escenario de conflicto en algunos profesorados de educación física de la CABA y otras localidades al momento de tramitar las prácticas docentes.

En cuanto a los recursos pedagógicos disponibles para la implementación de la ESI, la ausencia de representaciones de la diversidad sexo-genérica y corporal fue un obstáculo significativo. Algunas críticas a los recursos específicos -como a los cuadernos para la implementación de los contenidos curriculares de la ESI-señalaron que "la diferencia genital se presenta binaria sin considerar corporalidades gestantes como en el caso de los varones trans o personas intersexuales. Se profundiza la complejidad si se tiene en cuenta a las infancias trans en la escuela, siendo que muches niñes trans expresan su identidad en la socialización de la educación inicial" (Rueda, 2018, p. 257). ${ }^{18}$ Esta vacancia de recursos que posibiliten representaciones de la variabilidad de las corporalidades pone de manifiesto las tensiones entre diferentes epistemologías que o bien asimilan y/o promueven, o bien critican y/o resisten la normalización sexo-genérica binaria de lo humano.

Es elocuente la necesidad de incorporar otras nuevas perspectivas a la vez que, fundamentalmente, desarmar las estructuras tradicionales de inteligibilidad de los

\footnotetext{
18 Cabe aclarar que la mayoría de los recursos pedagógicos y didácticos elaborados por el Programa Nacional ESI del Ministerio de Educación de la Nación fueron publicados durante los primeros años posteriores a la sanción de la ley N²6.150, y que este trabajo citado (Rueda, 2018) plantea una lectura crítica de los materiales casi una década después, cuando otros cambios en los marcos normativos y los debates sociales aparejados ya estaban más instalados para revisar críticamente los enfoques asumidos en el marco de esta política pública.
} 
cuerpos sexuados. Esto ha significado la producción de lecturas críticas sobre las representaciones de la corporeidad que hasta el momento han circulado por las escuelas a través de textos y manuales escolares, láminas, revistas y cuadernillos del Programa de ESI, entre otros materiales didácticos. Los recursos oficiales disponibles resultaron insuficientes para incorporar la vasta transformación de los derechos ciudadanos adquiridos como parte de los marcos normativos posteriores a la sanción de las leyes de la ESI. Los tiempos de construcción de materiales didácticos, definición de los diseños curriculares y otros recursos pedagógicos generaron un hiato de sentidos entre las transformaciones epistémicas y los discursos tradicionales que sostuvieron un binarismo sexo-genérico.

Entre estas inflexiones de sentidos encontramos las profundas tensiones y disputas que se desplegaron por el uso del denominado "lenguaje inclusivo" como una de las formas de interpelar los modos dominantes del binarismo sexo-genérico en el sistema escolar, hilvanando pedagogías feministas, queer/cuir y trans para transformar los marcos epistemológicos que sustentaron el currículum escolar y conformaron, a su vez, las formas de habitar y transitar las instituciones educativas. Entendemos que este aspecto socialmente tan prolífico como asunto de debate en tan múltiples escenarios pone de manifiesto, aunque no resulta suficiente, las profundas modificaciones en las formas de nombrar en tanto mutación epistemológica de nuestros modos de comprender los cuerpxs, los sexos, los géneros, las sexualidades.

Entre otras voces en las que resuena el reclamo por la falta de representaciones en la escolaridad, Alba Rueda (2018) sostiene que, desde su diseño hasta su implementación, la ESI mantiene una deuda con la disidencia sexual, en general, y con la población travesti, trans, no binaria e intersex, en particular: hay vacancia de contenidos, pocas voces de personas TLGBQI+, ausencia de problematizaciones que expongan sus condiciones de vida y escolaridad, entre otras cuestiones. Históricamente, para aquellas personas que no se ajustaban al patrón sexo-genérico binario sus dificultosas experiencias de la escolaridad estuvieron fuertemente marcadas por su interrupción, dadas las condiciones expulsivas de las instituciones educativas que fueron relevadas por el estudio fundante La gesta del nombre propio. Informe sobre la situación de la comunidad travesti en la Argentina (2005) y recientemente actualizado en la investigación realizada por estudiantes y docentes del Bachillerato Trans Mocha Celis, La revolución de las mariposas (2017). Estos estudios contribuyen a lo que Rueda (2018) demanda como una necesidad de procurar una "memoria histórica" que, desde una perspectiva crítica profunda, problematice los modos en los que actuaron las escuelas y otras instituciones -como sindicatos, agrupaciones políticas partidarias y no partidarias, programas estatales logrados a partir de intervenciones de activistas feministas y TLGBQI+, entre otras- para garantizar, de esta manera, una ruptura con el status quo de las violencias institucionales.

Por su parte, la agenda del movimiento intersex está centrada en que se reconozca la diversidad de cuerpos sexuados con que nacen las personas y desarmar el sistema 
binario de normalización corporal que interviene, ajusta y "normaliza" características sexuales. En este caso nos interesa subrayar esta propuesta de poner el énfasis en la agenda de la diversidad corporal: desde un abordaje crítico, se concibe la intersexualidad centralmente como una cuestión de derechos sexuales (Cabral, 2009). Esta apuesta política también derrama en las invitaciones a repensar las pedagogías desplegadas en la ESI, particularmente al momento de abordar las corporalidades y el ejercicio de los derechos.

\section{La ESI frente a la despatologización de las identidades y las expresiones de género y las orientaciones sexuales}

En este proceso de transformaciones y en sinergia con esta fuerza epistémica, se va entroncando cada vez más el impacto de la pregunta y la reflexión por lo no binarie $^{19}$ en el campo pedagógico. Los géneros fluidos y no binaries como categorías políticas van traccionando nuevos sentidos sobre sexualidades, identidades de género y expectativas diferenciadas según el binarismo sexo-genérico imperante.

La ESI posibilitó en muchos contextos institucionales ir deconstruyendo esos andamiajes que eran tan nítidos como categóricos. El desafío no consiste meramente en normalizar los casilleros de varones homosexuales, mujeres lesbianas, personas bisexuales y personas trans, femeninas y masculinas, sino de hacer lugar también a esos intersticios que se vuelven la indefinición, que constituyen el borramiento mismo de las coordenadas y de las estructuras semánticas y los modos concretos de conceptualización existentes que están forjados sobre lo binario, incluso destrozando los tótems de lo binario: "lo macho", "lo hembra", "Lo varón", "Lo mujer", "Lo femenino" y "lo masculino". Sobre esos escombros de una epistemología interpelada, fisurada, se va (re)construyendo y (re)armando un lenguaje que empieza a vislumbrar lo no binario pero que, a la vez, nos lleva a otro campo de posibilidades no clausurado por sus formas polares.

Estos cambios epistemológicos y epistémicos impactaron institucionalmente en pos de una transformación de las prácticas pedagógicas concretas para garantizar otros existenciarios, otros modos de construir y vivir la corporalidad, otras prácticas de subjetivación, otras formas de narrarse a unx mismx. Pero este proceso fue tramándose de modo desigual y errático de acuerdo con los contextos institucionales, la discrecionalidad de las conducciones $y$, especialmente, desde las demandas

19 El lenguaje inclusivo recurre a diferentes modos de interrumpir la declinación binaria de la lengua castellana. Generalmente oscila entre el uso de la "x" y el uso de la "e". Si bien la "x" parece mostrar visualmente la cancelación de la lectura binaria tradicional, habitualmente en la oralidad se la pronuncia como "e". Aquí hemos usado intermitentemente ambas formas, aunque, sin embargo, se ha señalado que en los sistemas de lectura Braille puede resultar confusa la utilización de la "x". Más allá de esto, se ha observado un corrimiento hacia un uso más habitual de la "e" principalmente para referirse a y entre no binaries, pero también como modo de trascender la limitación de la x o el asterisco en la escritura, con gran impacto en el sistema educativo. 
docentes y estudiantiles para gestionar estas oportunidades.

Entre las cuestiones que fueron objeto de debate institucional, los cambios en las formas de organizar la vida escolar cotidiana $y$, por supuesto, los profundos desencuentros ocurridos, se fue instalando la problematización del nombre propio, la vestimenta y los códigos de convivencia, los juegos, la clase de educación física y su foco en la competencia deportiva sexuada, las filas separadas de nenas y nenes, los baños y otros protocolos escolares que redundaron en la regulación binaria de los géneros. En todas estas experiencias de la vida escolar se puso en evidencia que se van haciendo cada vez más corrientes las capas de sentido sedimentadas en los debates y las prácticas pedagógicas en consonancia con los activismos para la transformación institucional.

En el campo educativo, aquello implica revisar críticamente las prácticas de discriminación, asícomo reconocer laalta cuota desufrimiento que sigue gestionándose institucionalmente. Especialmente se trata de abordar estas cuestiones a través de la ESI en tanto tecnología reflexiva para la contención de los procesos emocionales, afectivos y vinculares que enmarcan y condicionan las experiencias de la escolaridad. En este enclave, las prácticas de acoso, hostigamiento y burla, que generalmente se conceptualizan como bullying, son constitutivas de los vínculos en general y deben ser reflexionadas como trama relacional de los modos de construcción de las masculinidades y las feminidades. Sin embargo, actualmente, la centralidad que han tomado los debates públicos y las políticas públicas contra las violencias de género fueron haciendo declinar el "fervor del macho" en pos de poner permanentemente en cuestión las masculinidades, exponiendo su crisis actual de hegemonía como género históricamente privilegiado por una diferenciación binaria desjerarquizante de lo femenino y todo lo que se corra de los cánones de la masculinidad hegemónica. Luego de décadas de una prolífica producción crítica sobre géneros y sexualidades desde diferentes campos disciplinares, que permitieron atender, visibilizar y comprender las relaciones de poder y las formas de subordinación de las mujeres y otras posiciones femeninas con respecto a los varones sobre la base de la construcción de estereotipos en el contexto de una sociedad patriarcal, los estudios sobre masculinidades tuvieron su impacto en los modos de promover también la reflexión sobre los procesos de construcción de relaciones jerarquizadas. Se produjeron, entonces, materiales pedagógicos en formato escrito y audiovisual que promovieron fuertemente lo que se denominó "la deconstrucción" de las masculinidades ${ }^{20}$.

El proceso de cambio epistemológico y epistémico interceptó la conformación de herramientas de la política educativa tales como documentos, resoluciones,

20 En el año 2012, se conformó una organización denominada Colectivo de Varones Antipatriarcales, entre CABA y otras regiones del país, con el propósito de aportar a la lucha contra el sistema de opresión basado en las asimetrías de poder entre los sexos. Tuvo una constante intervención en contextos académicos y educativos, generando espacios de reflexión en general y sobre situaciones concretas en diferentes instituciones. 
protocolos y guías para intervenciones institucionales que procuren la "inclusión", es decir, el acompañamiento de la trayectoria educativa del estudiantado para garantizar el acceso, la permanencia y la finalización de la escolaridad. Por su parte, algunas efemérides claves fueron incluidas en el calendario escolar como estrategia de visibilización de acontecimientos históricos y políticos ligados a las sexualidades y los géneros. ${ }^{21}$

\section{Conclusiones: la ESI como praxis política para la emancipación}

"Sería de suma importancia que se hiciera presente en la escuela la direccionalidad política que están marcando los movimientos sociales -en este caso, los movimientos sociosexuales-, que reclaman por una ciudadanía sexual plena y por el reconocimiento de géneros y sexualidades diversas." (Alonso et al., 2008, p. 271).

En este trabajo procuramos mostrar la sinergia entre los procesos de institucionalización a través de marcos normativos vinculados a la ESI, cambios epistémicos aparejados, (re)apropiaciones activistas de las leyes y los giros epistémicos y epistemológicos que se produjeron en el contexto educativo, presentando una compleja cartografía política de un movimiento pedagógico en expansión formado en torno a la ESI. La emergencia y la potencia de este movimiento pedagógico se fue desarrollando en relación con el proceso de institucionalización de la ESI como política pública. Este movimiento se nutre de la articulación con diversos modos de activismos, que se fueron multiplicando y repotenciando mutuamente con intervenciones políticas que exceden el espacio de las instituciones educativas. Todo ese fervor político, abonado principalmente por los movimientos feministas y de las disidencias sexo-genéricas, fue condicionando la institucionalización de la ESI. La ventana abierta por la ley 26.150 fue sin dudas una plataforma sustantiva durante este proceso de construcción de la política pública que se volvió clave para que las demandas activistas cobren mayor legitimidad hasta lograr la centralidad del eje de la diversidad en tanto valoración y perspectiva a asumir, redefiniendo epistémica y epistemológicamente las prácticas de la ESI.

Simultáneamente, durante el proceso recortado en este trabajo, se fueron

21 A modo de ejemplificación referimos la Ley Nº6.272 del año 2019, que instituyó en la CABA "Día de la Promoción de los Derechos de Las Infancias y Adolescencias Trans". Unos años atrás, otra incorporación en el calendario escolar traccionó los propósitos de visibilizar el "Día de Lucha Contra la Discriminación por Orientación Sexual o Identidad de Género" (Ley N²687, CABA), en conmemoración de la fecha en que la Organización Mundial de la Salud, en 1990, eliminó la homosexualidad de la lista de enfermedades mentales. Por su parte, la Colectiva Feminista La Revuelta incentivó que se aborden ciertas fechas para recrear la memoria histórica, como el 28 de junio en conmemoración de la revuelta de Stonewall (1969), en tanto oportunidades para contar otras historias, desde una perspectiva antidiscriminatoria, no androcéntrica, no sexista y no heterosexista, en el marco de la Jornada de Formación: "Por otras efemérides en la Educación Pública" (2010). La estrategia de instalar nuevas efemérides permitió generar nuevas oportunidades de reflexión e intervención institucional. 
legitimando progresivamente aquellas voces, perspectivas y propuestas provenientes de los distintos activismos, instalándose en e interpelando los espacios institucionales. Fueron zanjando nuevas (re)territorializaciones institucionales que, conjuntamente, a través de los ejes de la despatologización, la descriminalización y los derechos humanos, fueron impulsando y produciendo el giro epistémico y epistemológico para resituar las prácticas de la ESI. Desde estas nuevas perspectivas se van trazando otras ciudadanías legítimas posibles, que requieren no sólo más espacio simbólico sino también una revisión y reorientación de las condiciones institucionales de participación en la producción y circulación escolar de los conocimientos y los saberes que (pro)mueven la ESI.

Una consecuente posibilidad de formular preguntas más abiertas va instalándose en la apuesta político-pedagógica de la ESI, sobre todo cuando se requiere considerar los tránsitos, los intersticios, y no clausurar experiencias ni oportunidades en las escuelas. En este complejo campo de disputas se viene produciendo un reordenamiento de valores que estimulan la desestigmatización y la despatologización trans, travesti y no binarie en el marco de una ESI obligada a revisar el perimido concepto binario de persona humana cuya base de reconocimiento como tal sea su signo M o F. Este giro epistémico y las aventuras epistemológicas que supone abren horizontes políticos en las prácticas pedagógicas: despatriarcalizar, desandrocentrar, desheterocentrar, desciscentrar, desmisoginizar, deshomolesbotransbiodiar, desbinarizar y otres devenires.

\section{Bibliografía}

Alonso, G; Herczeg, G.; Zurbriggen, R. (2008). Talleres de educación sexual. Efectos del discurso heteronormativo. En Morgade, G.y Alonso, G. (Comp.) Cuerposy sexualidades en la escuela. De la normalidad" a la disidencia. (pp. 251-272). Buenos Aires, Argentina: Paidós.

Báez, J. (2013). La experiencia educativa "trans". Los modos de vivir el cuerpo sexuado de los/as/xs jóvenes en la escuela secundaria. (Tesis doctoral en Educación). Facultad de Filosofía y Letras. Universidad de Buenos Aires. Buenos Aires.

Barrancos, D. Prólogo. En Elizalde, S.; Felitti, K. y Queirolo, G. (Coords). (2009). Género y sexualidades en las tramas del saber. Revisiones y propuestas (pp. 11-14). Buenos Aires, Argentina: Libros del Zorzal.

Berkins, L. y Fernández, J. (coord.). (2005). La gesta del nombre propio. Informe sobre la comunidad travesti en la Argentina. Buenos Aires, Argentina: Editorial Madres de Plaza de Mayo.

Cabral, M. (Comp.) (2009). Interdicciones. Escrituras de la intersexualidad en castellano. Córdoba, Argentina: Anarrés Editorial, Proyecto de Mulabi, el Espacio Latinoamericano 
de Sexualidades y Derechos.

Colectivo Mariposas Mirabal (2019). Educación Sexual Integral. Epistemología, pedagogía y política en los debates curriculares. Cuadernos del IICE, 3. Facultad de Filosofía y Letras, Universidad de Buenos Aires.

La revolución de las mariposas (2017). A diez años de la gesta del nombre propio. Buenos Aires, Argentina: Programa de Género y Diversidad Sexual, la Fundación Divino Tesoro y el Bachillerato Popular Trans Mocha Celis. Ministerio Público de la Defensa de la Ciudad Autónoma de Buenos Aires.

Lavigne, L. (2015) Una etnografía sobre sexualidades, género y educación. La educación sexual integral en la Ciudad de Buenos Aires como política de gestión de la sexualidad juvenil (Tesis doctoral). Facultad de Filosofía y Letras, Universidad de Buenos Aires. Buenos Aires.

Lavigne, L. y Péchin, J. (En prensa). Cartografía crítica de la Educación Sexual Integral como enclave institucional: transmutaciones epistémicas y resistencias al binarismo sexo-genérico. En Álvarez, M. y Giamberardino, G. (Comps.). Ensamblajes de género, sexualidad(es) y educación. Intervenciones entre el activismo y la academia (s/pp.). Tandil, Argentina: Editorial UNICEN.

Mansilla, G. (2015). Yo nena, yo princesa. Luana, la niña que eligió su propio nombre. Buenos Aires, Argentina: Universidad Nacional de General Sarmiento.

Marcus, G. E. Etnografía Multisituada. Reacciones y potencialidades de un Ethos del método antropológico durante las primeras décadas de 2000. Etnografías Contemporáneas, 4(7).

Morgade, G. (Coord.). (2011). Toda educación es sexual. Buenos Aires, Argentina: La Crujía.

Noblit, G.; Flores, S. y Murillo, E. (2004). Post Critical Ethnography: Reinscribing Critique. New Jersey, USA: Hampton Press.

Péchin, J. (2012). Géneros, sexualidades y resistencias políticas a la normalización Etnografía crítica sobre procesos identitarios en/desde la escolaridad del siglo XXI en Buenos Aires. (Tesis doctoral en Educación). Facultad de Filosofía y Letras. Universidad de Buenos Aires. Buenos Aires.

Péchin, J. (2017). Entre lo queer y lo cuir: arte, política y críticas pedagógicas en Argentina. Revista Interalia. A Journal of Queer Studies, 7. Número especial: Entre otrxs. Perspectivas queer en el mundo hispánico/Among Others. Queer Perspectives in Hispanic World. Eds.: Rafael M. Mérida Jiménez, Jorge Luis Peralta y Łukasz Smuga. 
Rockwell, E. (2009). La experiencia etnográfica. Historia y cultura en los procesos educativos. Buenos Aires, Argentina: Paidós.

Rueda, A. (2018). La Educación Sexual Integral: indagaciones desde las agendas travestis trans. Debate Revista Mora, 25, 255-262.

Suess, A. (2014). Cuestionamiento de dinámicas de patologización y exclusión discursiva desde perspectivas trans e intersex. Revista de Estudios Sociales, 49, 128-143. 